\title{
Resultados a largo plazo del tratamiento del estesioneuroblastoma. Experiencia en Aragón (1981-2003)
}

\author{
P. Bueso, J. Lambea, R. Andrés, J. I. Mayordomo, J. Martínez, D. Isla, P. Escudero, A. Sáenz, E. Polo, R. Lara, \\ R. Lastra, E. Ortega, R. Escó, A. Tres
}

\section{Resumen}

- Propósito: el estesioneuroblastoma o neuroblastoma olfatorio es un tumor infrecuente. Se han publicado sólo un millar de casos en la literatura médica. La edad media de presentación es a los 50 años, no teniendo predilección por ningún sexo. Es un tumor de agresividad local con recidivas locales tardías. Se han descrito metástasis a distancia, frecuentemente pulmón y hueso. Presentamos nuestra experiencia en el manejo y tratamiento de este tipo de tumor.

- Material y métodos: entre 1981 y 2003, 8 casos de estesioneuroblastoma fueron diagnosticados en el Hospital Clínico Universitario de Zaragoza, un hospital terciario con 882 camas, que es referencia para radioterapia de alta energía en todo Aragón, por lo que con toda probabilidad, este número de casos corresponde al de diagnósticos en la Comunidad Autónoma de Aragón en ese período.

Cinco varones y 3 mujeres con una mediana de edad de 60 años (rango 49-82). Los síntomas más frecuentes a la presentación incluyeron: obstrucción nasal, epistaxis, anosmia, exoftalmus, edema palpebral y tumefacción local. El estadio de Kadish al diagnóstico fue: 3 pacientes estadio B, 2 estadio $C$ y 3 estadio D. Cirugía, radioterapia y quimioterapia fueron frecuentemente combinadas. En dos pacientes el tratamiento fue cirugía, sola en un paciente y en otro con radioterapia radical. Tres pacientes recibieron quimioterapia, sola en dos pacientes y combinada con radioterapia en el otro. Tres pacientes fueron tratados con radioterapia sólo.

- Resultados: dos pacientes están vivos sin enfermedad tras 87 y 108 meses del diagnóstico y uno más está actualmente en tratamiento. Cuatro pacientes murieron con progresión a los 6, 8, 38 y 63 meses del diagnóstico. Un paciente falleció por un segundo tumor a los 36 meses del diagnóstico.

- Conclusión: el control local es un requisito esencial para obtener supervivencias a largo plazo en el estesioneuroblastoma.

Palabras clave:

Estesioneuroblastoma. Neuroblastoma. Cirugía. Radioterapia. Quimioterapia.

Oncología, 2004; 27 (2):80-84

Servicio de Oncología Médica

Hospital Clínico Universitario "Lozano Blesa"

Zaragoza 


\section{Summary}

- Purpose: Esthesioneuroblastoma or olfactory neuroblastoma is an infrequent tumour with only one thousand cases reported in medical literature. Mean age at presentation is $\mathbf{5 0}$ years, and there is no sex predominance. It is a locally invasive tumour and late local relapses are frequent. Distant metastases have been reported (most frequent sites are lung and bone). We present our experience in management and treatment of this type of tumours.

- Material and methods: Between 1981 and 2003, 8 cases of esthesioneuroblastoma were diagnosed in the University Hospital of Saragossa, Spain, an 882 bed-tertiary hospital, which is a reference for high-energy radiotherapy in Aragon. So this number of cases likely represents the total number of cases diagnosed in Aragon during this period.

Sex was male in 5 and female in 3. Median age: 60 years (range 49-82). Most frequent symptoms at presentation were: nasal obstruction, nasal bleeding, anosmia, exophtalmos, palpebral edema and local swelling. Kadish stage at diagnosis: 3 patients stage B, 2 stage $C$ and 3 stage D. Surgery, radiotherapy and chemotherapy were frequently combined. In two patients the treatment was surgery, alone in one patient and combined with radical radiotherapy in the other one. Three patients received chemotherapy, alone in two patients and combined with radiotherapy in the other one. Three patients were treated with radiotherapy alone.

- Results: Two pacients remain alive and progression free at 87 and 108 months from the diagnosis and one more patient is on therapy. Four patients died with prognosis at 6, 8, 38 and 63 months from diagnosis. One pacient died of a second tumour 36 months from diagnosis.

- Conclusion: Local control is essential for long term survival in esthesioneuroblastoma.

Key words: Esthesioneuroblastoma. Neuroblastoma. Surgery. Radiotherapy. Chemotherapy.

\section{Introducción}

El estesioneuroblastoma o neuroblastoma olfatorio es un tumor infrecuente. Se han publicado aproximadamente un millar de casos en la literatura médica' desde que en 1924 fue descrito por Berger².

Originado en la mucosa olfatoria, se presenta con una frecuencia aproximada del 3\% de todos los tumores nasales ${ }^{3}$. Su presentación clínica mas frecuente es la epistaxis y obstrucción nasal.

La edad media de presentación es a los 50 años, no teniendo predilección por ningún sexo.

Es un tumor de agresividad local con recidivas locales tardías. Se han descrito metástasis a distancia, siendo su localización mas frecuente en pulmón y hueso.

La supervivencia global a los 5 años sería, para tumores localizados en fosa nasal, del $75 \%$, en los que afectan senos paranasales $60 \%$ y en los casos que afectan órbita, base del cráneo o cavidad nasal del $41 \% 4$.

Para su tratamiento se dispone de cirugía, radioterapia y quimioterapia. La selección de una modalidad terapéutica o la combinación de varias es controvertida dada la poca experiencia acumulada en la literatura médica.

La finalidad de este artículo es presentar nuestra experiencia en el manejo y tratamiento de este tipo de tumores.

\section{Material y métodos}

Entre 1981 y 2003 ocho casos de estesioneuroblastoma (Tabla I) fueron diagnosticados en el Hospital Clínico Universitario de Zaragoza, un hospital terciario con 882 camas, que es referencia para radioterapia de alta energía en todo Aragón, por lo que con toda probabilidad, este número de casos corresponde al de diagnósticos en la Comunidad Autónoma de Aragón en este período.

Características de los pacientes: cinco varones y 3 mujeres con una mediana de edad de 60 años (rango 49-82). Los síntomas mas frecuentes a la presentación incluyeron: obstrucción nasal, epistaxis, anosmia, exoftalmus, edema palpebral y tumefacción local. 


\section{P. Bueso y cols.}

TABLA I

\begin{tabular}{|c|c|c|c|c|c|c|c|c|c|}
\hline Caso & $\begin{array}{l}\text { Edad } \\
\text { (años) } \\
\text { sexo }\end{array}$ & Estadio & $\begin{array}{l}\text { Trat. } \\
\text { inicial }\end{array}$ & $\begin{array}{l}\text { Intervalo } \\
\text { libre } \\
\text { de recidiva } \\
\text { (meses) }\end{array}$ & $\begin{array}{l}\text { Tipo } \\
\text { recidiva }\end{array}$ & $\begin{array}{l}\text { Tratamiento } \\
\text { de recidiva }\end{array}$ & $\begin{array}{l}\text { Intervalo } \\
\text { libre }\end{array}$ & $\begin{array}{l}\text { Situacion } \\
\text { actual } \\
\text { de recidiva } \\
\text { (meses) }\end{array}$ & $\begin{array}{l}\text { Supervivencia } \\
\text { global } \\
\text { (meses) }\end{array}$ \\
\hline 1 & 55/ Varón & $D^{a}$ & $Q^{c}$ & 3 & No respuesta & & 3 & Muerto con PRG* & 8 \\
\hline 2 & 82/ Varón & $\mathrm{C}$ & RT & $87+$ & & & $87+$ & Vivo sin PRG. & $87+$ \\
\hline 3 & 80/ Varón & $D^{b}$ & RT & $108+$ & & & $108+$ & Vivo sin PRG. & $108+$ \\
\hline 4 & 56/ Mujer & B & Cirugía & 1 & Local & No & 1 & Muerto con PRG. & 6 \\
\hline 5 & 60/ Mujer & $D^{b}$ & RT & 10 & Distancia & $Q^{d}$ & 10 & Muerto con PRG. & 38 \\
\hline 6 & 49/ Varón & B & RT & 24 & Local & Cirugía & 24 & Muerto con PRG. & 63 \\
\hline 7 & 67/ Varón & C & RT & 36 & & & 36 & Muerto sin enfermedadf & 36 \\
\hline 8 & 44/ Mujer & B & $\mathrm{QT}^{\mathrm{e}}+\mathrm{RT}$ & $9+$ & & & $9+$ & Vivo sin enfermedad & $9+$ \\
\hline
\end{tabular}

QT: Quimioterapia. RT: Radioterapia

a Metástasis óseas. ${ }^{\mathrm{b}}$ Adenopatias cervicales. ${ }^{\mathrm{C}}$ Ciclofosfamida, doxorrubicina y vincristina.

${ }^{d}$ Ciclofosfamida, doxorrubicina, vincristina y cisplatino. Para la progresión: ifosfamida y VP-16.

e Cisplatino y VP-16 ${ }^{\mathrm{f}}$ Mieloma.

* PRG. : Progresión.

Para su estadiaje se emplea el propuesto por Kadish 5 y más tarde modificado por Morita ${ }^{6}$ : estadio A corresponde al tumor localizado en fosa nasal, B cuando afecta a senos paranasales, $C$ invade órbita, base del cráneo o cavidad nasal y $\mathrm{D}$ cuando existen metástasis a distancia e invasión de ganglios cervicales.

El estadio al diagnóstico fue: tres pacientes estadio $B$, dos estadio $C$ y 3 estadio $D$ (metástasis ganglionares cervicales en dos pacientes y en uno metástasis óseas).

\section{Tratamientos iniciales}

\section{Cirugía}

La cirugía fue practicada como tratamiento único en un paciente en estadio $B$ (caso 4 ) , incluyendo extirpación del tumor y maxilectomía.

\section{Radioterapia}

Cinco pacientes recibieron radioterapia como tratamiento inicial a dosis radicales (60-65 Gy): dos en estadio $D$, dos en $C$ y uno en $B$, tres de ellos como tratamiento único (casos 2, 3 y 7 ).

A un paciente en estadio $D$ (caso 5) posteriormente se le realizó quimioterapia.

El paciente número 8 se encuentra en tratamiento con cisplatino y etopósido, seguido de radioterapia.

\section{Quimioterapia}

Dos pacientes recibieron quimioterapia como trata- miento inicial, uno en estadio $D$ y otro en estadio $B$ (casos 1 y 8).

El paciente número 1 (estadio $D$ por metástasis costales al diagnóstico), recibió quimioterapia como tratamiento de primera línea con una combinación de Ciclofosfamida, Adriamicina y Vincristina por 4 ciclos, suspendiéndose por progresión de la enfermedad.

El paciente número 8 se encuentra en tratamiento con Cisplatino y Etopósido, seguido de radioterapia.

\section{Tratamientos de rescate}

\section{Cirugía}

El paciente número 6, tratado inicialmente con radioterapia, presentó al año de recibirla una recidiva local objetivada en tomografía axial computerizada de control. Por ello se practicó cirugía de rescate. A pesar de esto, el paciente presentó posteriormente progresión local no controlable y falleció.

\section{Quimioterapia}

El paciente número 5, tratado inicialmente con radioterapia presentó recidiva local y hepática 10 meses después de radioterapia, por lo que se le aplicó quimioterapia con Ciclofosfamida, Cisplatino, Adriamicina y Vincristina, con respuesta parcial seguida de progresión local y 3 ciclos de Ifosfamida, Etopósido, suspendiéndose por ausencia de respuesta. 


\section{Resultados}

\section{Supervivencia}

Los resultados de este estudio (Tabla I) muestran que el estesioneuroblastoma es un fumor agresivo. La mediana de intervalo libre de progresión es de 24 meses con 4 recidivas antes de los 12 meses del diagnóstico en los 8 pacientes. Pero el tratamiento locorregional radical puede conseguir supervivencias prolongadas. Así, dos pacientes están vivos sin enfermedad tras 87 y 108 meses del diagnóstico y un paciente falleció por un segundo tumor (mieloma múltiple) a los 36 meses. La mediana de supervivencia global es de 38 meses.

En los dos pacientes tratados mediante quimioterapia con Cisplatino se ha objetivado una respuesta parcial. No hubo respuesta en el tratado con quimioterapia sin Cisplatino.

\section{Discusión}

Desde que en 1924 Berger describió esta entidad, se han barajado diversas modalidades terapéuticas en los diferentes estadios de la enfermedad.

En estadios localizados $A \circ B$, las opciones se basan en la radioterapia y cirugía, solas o en combinación. En cuanto al tratamiento quirúrgico de los tumores, la técnica de elección hasta el momento sería la resección tumoral por vía craneofacial ${ }^{5}$. Respecto a la combinación de radioterapia y cirugía, un estudio realizado en la clínica Mayo entre 1951-1990, muestra que no existen diferencias significativas entre la supervivencia de los pacientes tratados con radioterapia adyuvante y los que solamente se les realizó cirugía ${ }^{7}$; otros autores como Elkon objetivan recurrencias de la enfermedad en el $50 \%$ de pacientes estadio $B$ tratados con cirugía exclusivamente, frente al $40 \%$ de pacientes que en igual estadio recibieron tratamiento combinado ${ }^{6}$. Broich, en una revisión de los casos publicados desde 1924, indica que la mortalidad era más elevada en aquellos pacientes que solamente recibieron tratamiento radioterápico, siendo de $30,77 \%$ frente a $18,75 \%$ en los que recibieron tratamiento combinado'. Schwaab encuentra una supervivencia a los 5 años de $56 \%$ para los pacientes tratados con cirugía más radioterapia, frente a $26 \%$ para los que fueron tratados solamente con radioterapia ${ }^{8}$. En un meta-análisis realizado por Dulguerov en 2001 que comprende los casos publicados entre 1990 y 2000, se llega a la conclusión de que el tratamiento óptimo es la combinación de radioterapia y cirugía, obteniendo una supervivencia del $65 \%$ frente al $48 \%$ y $37 \%$ obtenidas con radio- terapia y cirugía solas, respectivamente 9 . Theilgaard, en 2003, en un estudio sobre 40 pacientes, defiende el tratamiento combinado quirúrgico y radioterápico en estadios $A$ y $B$, mientras que en el $C$ debe tratarse además de con cirugía y con radioterapia, con quimioterapia $^{10}$.

Respecto a las dosis de radioterapia, dado que es un tumor con comportamiento agresivo y con tendencia a la recidiva local, las dosis recomendadas son radicales (60-65Gy). Guedea demuestra control local de la enfermedad en una serie de 7 pacientes con enfermedad localmente avanzada, tratados todos ellos con dicha dosis de radioterapia ${ }^{11}$. En la serie de Ahmad ninguno de los pacientes que recibieron dosis radicales de radioterapia recidivaron localmente ${ }^{12}$.

La quimioterapia puede jugar un papel en estadios localmente avanzados (estadio C) o metastásicos (estadio D). Desde 1950 ha sido utilizada. Uno de los primeros fue Mendeloff, en 1957, utilizando mostaza nitrogenada concomitante con radioterapia ${ }^{13}$. Posteriormente se han utilizado agentes simples con experiencia anecdótica, incluyendo Thiotepa, Doxorrubicina, Ciclofosfamida, Vincristina, DTIC y mostaza nitrogena$\mathrm{da}^{14}$. Otros esquemas han incluido combinaciones de Ciclofosfamida, Vincristina y Adriamicina ${ }^{15}$. Wade objetivó que el mayor número de respuestas se consigue tras tratamiento con Ciclofosfamida y Vincristina ${ }^{12}$. Morita describe su serie de 49 pacientes tratados con quimioterapia, combinada con quimioterapia precoz en estesioneuroblastoma localmente avanzado o de alto grado tumoral, con supervivencias a los 5 años del $40 \%$. Spaulding combina distintas modalidades de tratamiento en pacientes estadio $\mathrm{C}$, realizando 2-3 ciclos de quimioterapia, esquema Ciclofosfamida + Vincristina seguido de radioterapia (dosis $50 \mathrm{~Gy}$ ) y posterior resección craneofacial, pero no existían diferencias significativas con los resultados de controles histó$\operatorname{ricos}^{16}$. En un estudio alemán se concluye que en estadio $C$, la mejor alternativa es la combinación de cirugía, radioterapia y quimioterapia, previniendo las recidivas locales y sistémicas y aumentando la supervivencia ${ }^{17}$. La quimioterapia a altas dosis también ha sido utilizada ${ }^{18-20}$ aunque a nuestro parecer sería un tratamiento demasiado agresivo en este tipo de tumor en el que la quimiosensibilidad está aún en estudio. Nuestra experiencia es que los dos pacientes tratados con cisplatino junto a otros fármacos, obtuvieron una respuesta parcial, mientras que el paciente que no lo recibió no respondió al tratamiento quimioterápico.

En conclusión, el estesioneuroblastoma es un tumor raro aunque no infrecuente, para el que se dispone de terapéuticas que permiten el control local en una alta proporción de casos. La radioterapia combinada 


\section{P. Bueso y cols.}

con cirugía, sería un tratamiento eficaz en los estadios más localizados: $A$ y $B$. La quimioterapia se podría incluir en el tratamiento combinado de aquellos pacientes en los que sea más difícil el control, bien por su extensión local o por su condición de metastásico, aunque su efectividad todavía requiere investigaciones posteriores.

Correspondencia:

Dr. J. I. Mayordomo

Servicio Oncología Médica

Hospital Clínico Universitario "Lozano Blesa"

Avenida San Juan Bosco, 15

E-50009 Zaragoza

josemayordomo@hotmail.com

\section{Bibliografía}

1. Broing G, Pagliari A. Ottavian F. Esthesioneuroblastoma: a general review of the cases published since the discovery of the tumor in 1924. Anticancer Reseach 1997; 17:2683-706.

2. Berger L, Luc R. L'esthesioneuroepithelioma olfactif; Bull de l'assoc franc pour l'etude du cancer (Paris) 1924; 13: 410-20.

3. Mc Cormack $\sqcup$, Harris HE. Neurogenic Tumors of the nasal fossa. Jama 1955; 157:318-21.

4. Elkom D, Hightower SF, Lim ML, et al. Esthesioneuroblastoma. Cancer 1979; 44:1087-94.

5. Kadish S, Goodman M, Wang CC. Olfactory Neuroblastoma. Cancer 1976; 37:1571-5.

6. Morita A, Ebersolod MJ, Olsen KD, et al. Esthesioneuroblastoma: prognosis and management. Neurosurgery 1993; 32:706-15.

7. Foote RL, Morita A, Ebersold MJ, et al. Esthesioneuroblastoma: the role of adjuvant radiation therapy. Int. J. Radiat. Oncol Biol Phys 1993; 27:835-43.

8. Schwaab G, Lefebvre JL, Julieron M. Cystic adenoid car- cinomas and olfactory esthesioneuromas of the ORL group of the National Federation of Cancer Centers. Neurochirurgie 1997; 43(2): 118-20.

9. Dulguerov $P$, Allal AS, Calcaterra TC. Esthesioneuroblastoma. Lancet Oncol. 2001 Nov; 2(11):683-90

10. Theilgaard SA, Buchwald C, Ingeholm P, Kornum Larsen S, Eriksen J, San Hansen H. Esthesioneuroblastoma: a Danish demographic study of 40 patients registered between 1978 and 2000. Acta Otolaryngol. 2003; 123(3): 433-9.

11. Guedea F, Van Limbergen E, Van Den Bogaert W. High dose level radiation therapy for local tumour control in Esthesioneuroblastoma. Eur. J. Cancer 1994; Vol 30ª , 12:1757-60.

12. Ahmad K, Fayos JV. Role of radiation therapy in the treatment of olfactory neuroblastoma. Int. J. Radiat. Oncol. Biol. Phys 1980; 6:349-52.

13. Mendeloff J. The olfactory neuroepithelial tumors. Cancer 1957; 10:944-56.

14. Wade PM, Smithre Johns ME. Response of Esthesioneuroblastoma to chemoterapy: report of five cases and review of the literature. Cancer 1984; 53:1036-42.

15. Walters TR, Pushparaj N, Ghander AZ. Olfactory neuroblastoma: response to combination chemotherapy. Arch. Otolaryngol. 1980; 106:242-3.

16. Spaulding C, Kranyak MS, Constible WC, Stewart FM. Esthesioneuroblastoma: a comparison of two treatment eras. Int. J. Radiation Oncology Biol Phys. 1988; 15. 581-90.

17. Eich HT, Hero B, Staar S et al. Multimodality therapy including radiotherapy and chemotherapy improves eventfree survival in stage $C$ esthesioneuroblastoma. Strahlenter Onkol. 2003; 179(4):233-40.

18. O'Conor gt, Drake CR, Johns ME, et all. Treatment of advenced esthesioneuroblastoma with high-dose chemotherapy and autologous bone marrow transplation. A case report. Cancer 1985; 55:347-9.

19. Stewart FM, Lazarus HM, Levine PA, et all. High-dose chemotherapy and autologous marow transplation for esthesioneuroblastoma sinonasal and undifferentiated carcinoma 1989; 12(3):217-21.

20. Bernard V, Eden MD, Richard F, et all. Esthesioneuroblastoma long term outcome and patterns of failure the University of Virginia experience. Cancer 1994; 73: 2556-63. 